https://doi.org/10.15407/ujpe64.1.48

B.I. LEV, A.G. ZAGORODNY

Bogolyubov Institute for Theoretical Physics, Nat. Acad. of Sci. of Ukraine

(14b, Metrolohichna Str., Kyiv 03680, Ukraine)

\title{
COLLECTIVE DIFFUSION OF COLLOIDAL PARTICLES IN A LIQUID CRYSTAL
}

\begin{abstract}
The theory of collective diffusion effects in a system of colloidal particles in a liquid crystal is proposed. The specifics of diffusion which can be observed experimentally are described. The dependence of the diffusion coefficient on the temperature and particle density is found. It is shown that collective diffusion in a system of colloidal particles in a liquid crystal arises from the elastic distortion of the director field generating the interparticle interaction. The behavior of such diffusion is found to be nontrivial.

Ke ywords: diffusion, liquid crystalls, colloidal particles, collective interactions.
\end{abstract}

Recently much interest has been generated to the studies of colloidal particles in liquid crystals. Anisotropic properties of the host fluid of a liquid crystal give rise to a new class of colloidal anisotropic interactions that never occur in isotropic hosts. Liquid crystal colloidal systems also attract attention as models for various phenomena in condense matter physics. Anisotropic interactions generate different structures of colloidal particles such as linear chains in inverter nematic emulsions [1-4], 2D crystals [5], and $2 \mathrm{D}$ hexagonal structures at nematic-air interfaces [6]. The authors of [7] have observed 3D crystal structures in a system of hard particles with dipole configuration deformation of the director field. Recently [8] it was found that anomalous diffusion of an individual colloidal particle occurs at time scales that correspond to the relaxation times of the director deformations around the particle. Once the nematic melts, the diffusion becomes normal and isotropic. It was shows that deformations and fluctuations of the elastic director influence the diffusion modes and that sub-diffusion and super-diffusion can be observed. In this article we study the collective behavior of the

(C) B.I. LEV, A.G. ZAGORODNY, 2019

48 diffusion resulting from of particle interaction that can lead to the formation of new spatially nonuniform distributions [9-11]. Nontrivial behavior of the collective diffusion of colloidal particles in a liquid crystal is predicted. We show that the temperature dependence of the diffusion coefficient for various concentration is non-monotonic. It can be explained taking into account the specific properties of particle interaction. In the case of colloidal suspensions the diffusion coefficients usually are studied experimentally using the dynamic light scattering [12-15]. If one extrapolates the diffusion coefficient to a vanishing concentration of particles, it reduces to the single-particle diffusion coefficient since interactions between particles become negligible. On the contrary, for non-vanishing concentration particle interactions can influence the diffusion.

Complex studies of phase transitions, collective diffusion, autocorrelation function in this case allow to describe a new physical picture of the collective behavior in a colloidal medium that occurs in any liquid crystal. The critical non-monotonic temperature dependence of the viscoelastic response functions is associated with the long-range interaction between colloidal particles inducing the deformation of the

ISSN 2071-0194. Ukr. J. Phys. 2019. Vol. 64, No. 1 
elastic director field. Both temperature and concentration dependences of the viscoelastic response of a suspension of spherical colloidal particles are studied in the mean-field approximation in the vicinity of the isotropic-nematic transition. The explicit expressions for temperature and concentration dependences of the static structure factor are derived. Macroscopic expression for the anomalous part of the collective diffusion coefficient is obtained. It is shown that the temperature dependence of the drag force in the system of colloidal particles can be responsible for the nontrivial behavior of the collective diffusion in a liquid crystal. Colloidal liquid crystals are systems with structural and time scales that provide a possibility for the system to be non-equilibrium, or some exotic states during rather long time. Here, we focus our attention on the diffusion motion which can form such states. The rheologic properties of liquid crystals and the structure of phase-separating (size, shape of patterns and their spatial distribution) strongly affect the physical properties of colloids. Recently these aspects have been extensively studied that resulted in considerable progress in understanding of the relations between structures and rheologic properties. It is expected that the breakdown of the rotation symmetry may induce optical and mechanical properties. The flow of a liquid crystal around a particle depends on its shape and the viscosity coefficients and also on the direction of the molecule orientation [8]. The estimates of particle mobilities in liquid crystal obtained from the Stokes friction coefficient disagree with relevant experimental data. A probable reason for such a discrepancy may be the increase of the effective particle mass due to the existence of the deformation coat that moves together with the particle. Colloidal particle interaction can also make the reason for the friction enhancement [1-9]. The physical mechanism of this interaction is that the particle distorts the director distribution at the distances much longer than the particle size and thus provides an effective interaction with another similar particle by means of the elastic field deformation. The role of this interaction between colloidal particles is dual. First of all, it influences the viscoelastic response function. These macroscopic expressions are obtained by averaging the phase function over the ensemble, the elastic interaction function included. These long-range interaction functions are responsible for the strong diver- gence of the viscoelastic response function for colloids. Moreover, the ensemble average representing the viscoelastic response function should be evaluated with regard to the shear rate distorted paircorrelation function. The shear rate dependence of this probability density function is the result of the interplay between equilibrium restoring forces and shear forces. The anomalous contribution is associated with the divergent part the viscoelastic response function due to the long-range correlation induced by the particle interaction with the field of the director deformation. It should be noted that studies of the rheologic properties of colloidal liquid crystal suspensions are rather numerous [16-20]. Therefore, we can compare our results with those for the Newtonian viscosity. The macroscopic evaluation of the viscoelastic functions consists of the following steps: a) description of the mesosphere structure with the formation of a deformation coat solvate shell around the ordinary particle; b) the second step consists in the study of the influence of the director field elastic deformation on the particle interaction; c) the last step consists in the description of the probability phase transitions which are accompanied by the formation of a new structure observed experimentally.

The approach proposed in this paper makes it possible to describe the collective diffusion in liquid crystals for a wide range of temperatures and concentrations. We employ the concept of the effective mass and friction drag for a colloidal particle moving in a liquid crystal. As is shown in Ref. [9], the director field deformation moves together with the moving particle in spite of the extremely weak anchoring on the surface. The first, one deals with a spherical particle whose anchoring on the surface is strong $[21,24]$. It creates a topological defect in the vicinity of the particle that is necessary to satisfy the topological global boundary conditions. A particle with strong planar anchoring creates a pair of topological defects, known as boojums. On the other hand, a particle with strong homeotropic boundary conditions creates an equatorial disclination ring or a hyperbolic hedgehog as a companion for the radial hedgehog on the surface of the particle. Lubensky et al. [21] have applied the variation technique and the electrostatic analogy and thus obtained an approximate director distribution near the particle with normal boundary conditions, as well as the long-range pair interparticle interaction potential. 
The second approach was proposed in Ref. [9], the authors have examined the case of weak anchoring for particles of arbitrary shapes. They have found analytically the pair interaction potential taking into account different Frank constants and have expressed the potential in terms of the tensor with regard to the particle shape. In Ref. [11] it was shown that the long-range interaction potential between particles in a liquid crystal is determined by the symmetry breaking of the director field in the vicinity of the particle. This symmetry breaking is generated by two reasons: the shape of the particle and the anchoring. In the case of weak anchoring it is determined primarily by the particle shape. In the case of strong anchoring, on the other hand, both factors are essential because the director distribution near the particle is in this case determined by the topological defects in its vicinity. In order to give a general description of all these phenomena, the concept of the deformation coat around the particle was proposed. The deformation coat embraces all the accompanying topological defects and has symmetry similar to that of the resultant director field near the particle. The director distribution outside the coat undergoes only smooth variations and does not contain any topological defects. This colloidal particle may also be regarded as a microparticle surrounded by a solvate shell provided the interaction between such a particle and the molecules is much stronger than the intermolecular interaction responsible for the liquid crystal formation. The solvable formation may be regarded as a macroparticle, thus its interaction with another similar formation can be described in terms of the director field deformation. Taking into account the director distribution around an individual particle, one can find the change of orientation of the director induced by two particles and determine the change of deformation energy with separation of the energy component associated with the interaction between the particles. The case when the interaction of an individual particle with the director field deformation produced by other particles is determined by the anchoring on the surface of this particle is described in Ref. [9]. For the case when the number of particles is small and anchoring on the surface is weak (colloidal particles in a liquid crystal), the distribution of the director field is found in Ref. [17]. Let us describe this approach in brief. The strong anchoring directly implies that near the colloidal surfaces significant spa- tial variations of the director occur and even defects can appear. This means that the assumption about a roughly uniform director $\mathbf{n}=\left(n_{x}, n_{y}, 1\right)$ is no longer valid for the whole liquid crystalline volume. In order to explain the speculations in what follows we consider the case of a homogeneous liquid crystal with uniform director $\mathbf{n}_{\mathbf{0}}$ and one particle immersed into it. Anchoring of the liquid crystal with the surface produces particle deformations of the director field around the particle, so the director $\mathbf{n}(\mathbf{R})$ varies from point to point. In the one-constant approximation the total free energy of the system is given by

$F=\frac{K}{2} \int d V\left[(\operatorname{div} \mathbf{n})^{2}+(\operatorname{rot} \mathbf{n})^{2}\right]+\oint d S W(\nu \cdot \mathbf{n})^{2}$

where $W$ is the anchoring coefficient, $\nu$ is the unit normal vector, integration $\oint d S$ is carried out over the surface of the particle, $K$ is the Frank elastic constant. Far from the particle director field variations are small, $\mathbf{n}(\mathbf{R}) \approx\left(n_{x}, n_{y}, 1\right),\left|n_{\mu}\right| \ll 1(\mu=x, y)$, and the bulk free energy is given by

$F_{b, \text { linear }}=\frac{K}{2} \int d V\left\{\left(\nabla n_{x}\right)^{2}+\left(\nabla n_{y}\right)^{2}\right\}$,

which yields the Euler-Lagrange equation of the Laplace type, i.e.,

$\Delta n_{\mu}=0$.

At large distances $R$ in the general case it can be expanded in multiples, i.e.,

$n_{\mu}(\mathbf{R})=\frac{q_{\mu}}{R}+\frac{\mathbf{p}_{\mu} \mathbf{u}}{R^{2}}+3 \frac{\mathbf{u}: \hat{Q}_{\mu}: \mathbf{u}}{R^{3}}$

with $u_{\alpha}=R_{\alpha} / R ; \mathbf{p}_{\mu} \mathbf{u}=p_{\mu}^{\alpha} u_{\alpha}, \mathbf{u}: \hat{Q}_{\mu}: \mathbf{u}=Q_{\mu}^{\alpha \beta} u_{\alpha} u_{\beta}$, and $\mu=(x, y)$. This is the most general expression for the director field. We note that the multiple expression does not depend on the anchoring. It is valid for far distances for any anchoring, both weak and strong, either without topological defects or with them. Of course in order to find multiple coefficients we have to solve the problem in the near nonlinear area either with computer simulation or in terms of Ansatz functions. Suppose we have found all multiple coefficients for the particular particle (for instance with computer simulation). After this presentation we can use both approaches to describe 
the interaction energy that does not depend on the anchoring value on the particle surface. For particles with strong anchoring it is the far-region because director deformations in the near-region are strong. For particles with weak anchoring, however, distortions are small everywhere and the multiple expansion is applicable in the near-region too. The symmetry of the coat is equivalent to the broken symmetry of the director in the vicinity.

A way to avoid strong deformations of the director field and incorporate them in the analytical description is to introduce a "coat region" [11]. This region acts as an effective colloid which incorporates all strong deformations of the director field around the real particle and has the symmetry similar to the director distribution. In Ref. [22] the general paradigm of the elastic interaction between colloidal particles in a nematic liquid crystal was proposed. It implies that each every particle with strong anchoring and radius $R$ has is surrounded by three zones. The first zone for $r<1.3 R$ is the zone of topological defects, and in order to find the distribution of the director field we have to employ a nonlinear equation; the second zone in the approximate range $1.3 R<r<4 R$ is the zone where crossover from topological defects to the main multiple moment takes place. The last third zone is the zone of the main multiple moment, where higherorder terms may be disregarded. The explanation of this presentation is rather simple. In order to understand it and to find the dimensions of these zones we propose the procedure given below.

In order to find the director distribution around the spherical particle we have to minimize the Frank energy and take into account the boundary condition. This task was solved in Ref. [23]. In spherical coordinates we can take

$\mathbf{n}(\mathbf{r})=(\sin \beta \sin \gamma, \sin \beta \cos \gamma, \cos \beta)$,

where $\beta$ is the polar angle and $\gamma$ is the azimuthal angle. In this representation there occurs azimuthal symmetry with respect to $z$. The difference equation in the one-constant approximation reduces to

$\nabla \beta(r)-\frac{\sin 2 \beta(r)}{2 r^{2} \sin ^{2} \theta}=0$

and the boundary condition on the surface of the particle is given by

$\frac{\partial \beta}{\partial r}+\left.\frac{\beta}{r}\right|_{r=R}=-\frac{W}{2 K} \sin \theta$.

ISSN 2071-0194. Ukr. J. Phys. 2019. Vol. 64, No. 1
Its general solution is $\beta=\sum_{k} \frac{C_{k}}{r^{k+1}} P_{k}^{1}(\cos \theta)$ where $P_{k}^{1}(\cos \theta)$ is associated with the Legendre polynomial. The boundary condition selects the solution that in the case of weak anchoring $(R W / K) \leq 1$ is given by [24]:

$\beta(r)=(R W / 4 K) R^{3} \sin 2 \theta / r^{3}$,

where $W$ is the anchoring energy and $R$ is the radius of the spherical particle. In the case of small deformation of the director field $(R W / K)<4$. This relation determines the condition of week anchoring. In the case of strong anchoring $\left(R_{0} W / K\right) \geq 1$ and there appears a disclination ring of radius $a$ around the spherical particle. This solution was also obtained in Ref. [23]. It is given by

$\beta(r)=\theta-\frac{1}{2} \arctan \frac{\sin 2 \theta}{\cos 2 \theta+\left(\frac{a}{r}\right)^{3}}$.

Inside the disclination ring the solution for the director field distribution reduces to $\beta=\left(\frac{a}{r}\right)^{3} \sin 2 \theta$ where $a$ is the ring radius that may be obtained from the minimum of free energy. The estimation of the ring radius follows from the expression $a=\frac{5}{4} R$. For the disclination ring around the spherical particle to disappear, the anchoring associated with this solution must be similar to that in the case of week anchoring. Therefore we restrict the consideration to the qualitative estimate with the crossover value $W^{*} \sim \frac{4 K a^{3}}{R^{4}}=\frac{125}{16} \frac{K}{R}$. In this case the disclination ring around the spherical particle does not appear. This approach has been also applied to obtain the free energy which introduces a single particle in the liquid crystal $[21,24]$. In the case of week anchoring $F \approx \frac{W^{2} R^{3}}{5 K}$ while in the case of strong anchoring $F \approx 13 K R$. The uniform director distribution far from the particle has zero topological charge and so there should be another topological defect near the particle to compensate the hedgehog in the center. Obviously, the director configurations have different symmetries. The nonequatorial disclination ring and the pair of radial and hyperbolic hedgehogs break the mirror symmetry in the horizontal plane while the equatorial disclination ring (Saturn-ring) retains it. The authors of [24] have shown by Monte-Carlo simulations that the configuration with a hyperbolic hedgehog has lower energy, than Saturn-ring. It has been confirmed in [21] with the use of the dipole Ansatz that though the equatorial ring has some 
metastability, its energy is higher than the energy of the dipole configuration.

We can introduce the deformation coat which includes all strong deformations of the director field. This deformation area is a new "immersed particle" and we can use only self-consistence approach in the case of week anchoring and small deformation of the director field inside this new inclusion. We can estimate the size of this deformation coat if we take into account the total energy introduced in the liquid crystal by a particle. For a spherical particle of radius $R$ in the case of strong anchoring, the free deformation energy can be presented in the form [21]

$F^{\text {strong }} \approx \frac{9}{2} \pi K R$.

We assume that around the spherical particle there exists a Saturn-ring disclination. The size of the deformation area is denoted as $R^{*}$. At the next step we calculate the free energy which can introduce the deformation area inside. This energy can be obtained if we suppose that within this deformation area we have the case of week anchoring and can use the distribution director field as in the case of weak anchoring. This energy was obtained in Ref. [24], we have

$F^{\mathrm{weak}}=\frac{\pi}{15} \frac{W_{c}^{2} R^{* 2}}{K}$

where $W_{c}$ is the critical value of the anchoring energy for the case of different particle sizes when outside the particle singularity in the distribution of the director field does not appear. This critical value was also obtained in Ref. [24] and is given by $W_{c}=\frac{4 K a^{3}}{R^{4}}$ where $a$ is the radius of the Saturn-ring disclination around the spherical particle. Inside the deformation area there occurs a disclination ring. The free energy of this Saturn-ring disclination may be written in the form [21]

$F^{\mathrm{disc}} \approx 2 \pi K a\left(\frac{3 \pi}{4}+1\right)+8 \pi K\left(R^{*}-a\right)$,

where the size of the Saturn-ring can be written in the same form as before. To estimate the size of the deformation coat, we compare the free energy that really creates an inclusion in the case of strong anchoring to the sum of the free energy that creates the deformation coat outside and the free energy of the Saturn-ring disclination inside this coat. Within the context of the relation

$F^{\text {weak }}+F^{\text {disc }}=F^{\text {strong }}$,

the size of the deformation area reduces to $R^{*} \approx 2 R$. This estimation was made in the case of a Saturnring disclination inside the deformation coat, but this approach can be also used in the case of different defects which can appear inside this coat. In the case of a dipole configuration in the distribution of the director field inside this area estimation is correct to, but the deformation coat has asymmetric shape. The deformation coats in all cases have approximately equal sizes.

If the size of the elastic deformation is known, we can study the drag force and inertial particle characteristics. The friction drag force acting on a particle that moves in a nematic liquid crystal is known from the computer simulations $[17,19]$. We focus our attention on the effective mass of a particle moving in a liquid crystal. As was shown earlier, the motion of a particle traveling in a liquid crystal is accompanied by the motion of the director deformation field produced by the surface anchoring. Thus, a particle moving inside a nematic liquid crystal has the kinetic energy $T=m u^{2} / 2$ that contains the mass of the deformation coat $[11,18]$. We assume that the deformation coat adiabatically follows the moving particle; a similar assumption was used in constructing the polaron theory. In this regard it should be noted that this assumption implies the restriction on the particle velocity, $u \leq \tilde{R} t_{0}$, where $t_{0}$ is the time of the director transition to the steady state. relevant estimates of a spherical size $\tilde{R}$ of the deformation coat. In the framework of the concept of deformation coat, the director distribution around a particle is given by $\mathbf{n}=\mathbf{n}\left(t-\int u\left(t^{\prime}\right) d t^{\prime}\right)$. The numerical estimate of the effective mass can be obtained if the director distribution around the moving particle is known. In the case of weak anchoring [17] the isotropic part of the effective mass can be written as

$m_{\mathrm{eff}}=m+\frac{4 I}{3}\left(\frac{W}{4 K}\right)^{2} R^{3}$

in accordance to the results presented in Ref. [18], where $I$ is the density of the nematic liquid crystal moment of the inertia.

We have already shown that a foreign particle produces liquid crystal distortion in a region much larger

ISSN 2071-0194. Ukr. J. Phys. 2019. Vol. 64, No. 1 
than the particle size and thus leads to an effective interaction with another similar particle via the director field deformation. In this sense, the interaction of spherical particles is also associated with the director elastic field deformation. The particles dispersed in the liquid crystal cause long-range deformation of the director field. The self-consistent approach provides a possibility to find the energy of the inter-particle interaction. Then we can study the thermodynamic behavior of complexes of such particles and describe the conditions for the formation of new structures $[9,10]$. The properties of the interparticle interaction in the system of foreign particles in a liquid crystal determine the conditions of temperature and concentration phase transitions that accompany the formation of spatially inhomogeneous particle distributions $[17,25]$. The first-order phase transition can occur when the external field is present and introduces topological defects in the nematic ordering. Since the interaction between colloidal particles is a long-range, it can lead to strong critical divergence of the shear viscosity. Therefore, it is interesting to study the complete temperature dependence of the shear viscosity near the critical point when the phase transition is accompanied by the formation of inhomogeneous distributions in the system of colloidal particles.

It turns out to be that the viscoelastic response function contains two additive contributions associated with anomalous and background effects. The anomalous contribution is the part of the viscoelastic response function that diverges at the critical point due to the presence of long-range correlation. Thus the background contribution should be subtracted from the experimental viscoelastic response function in order to obtain the observable anomalous contribution, which may then be compared to the theoretical predictions. This means that the comparison of our results with the experimental data would be possible for the Newtonian viscosity, except for the case of essential difference in the inter-particle potential.

A fairly detailed description of the distribution of viscoelastic properties in the system of interacting particles is is given in Refs. [12-15]. Specifically, the estimate for $D_{\text {eff }}(\mathbf{k})$, where $k$ is the wavevector, is given by

$D_{\mathrm{eff}}=\frac{D}{k T}\left\{\frac{d G}{d \widetilde{\rho}}+q^{2} S\right\}$,

ISSN 2071-0194. Ukr. J. Phys. 2019. Vol. 64, No. 1 where $D$ is the ordinary diffusion coefficient for a spherical particle moving in condensed matter. The first term in the right-hand part of this equation describes the shear flow distortion of the interacting Brownian particle and can be written as

$G=\widetilde{\rho} k T-\frac{2 \pi}{3} \widetilde{\rho}^{2} c(1-c) \int d r^{\prime} r^{\prime 3} \frac{d V\left(r^{\prime}\right)}{d r^{\prime}} g^{e q}\left(r^{\prime}\right)$,

where $\widetilde{\rho}$ is the concentration of the medium, $c$ is the friction parameter for the particle that is foreign in the liquid crystal, $V\left(r^{\prime}\right)$ is the pair interaction energy between particles through the elastic deformation director field, and $g^{e q}\left(r^{\prime}\right)$ is the pair correlation function. The last term in Eq. (8) describes the diffusion contribution to the recovery of the equilibrium structure with

$S=\frac{2 \pi}{15} \widetilde{\rho} c \int d r^{\prime} r^{\prime 5} \frac{d V\left(r^{\prime}\right)}{d r^{\prime}} \times$

$\times\left\{g^{e q}\left(r^{\prime}\right)+\frac{1}{8} \widetilde{\rho}(1-c) \frac{d g^{e q}\left(r^{\prime}\right)}{d r^{\prime}}\right\}$.

Close to the critical point and also close to the offcritical part of the spinodal decomposition, which accompanies the first-order phase transition,, where $\frac{D}{k T} \frac{d G}{d \widetilde{\rho}}$ is small, the effective diffusion coefficient is small for small wave vectors, a phenomenon that is commonly referred to as critical slowing down.

As will be show, the diffusion coefficient can be explicitly related to the structure factor in two simple cases: weak inter-particle interaction through the director field deformation of the shear flow, and under stationary shear flow in the case of inter-particle interaction generating the phase transition with formation of spatially inhomogeneous particle distributions. The above equation is related to the mean-field description of the structure factor. It can be written in the form;

$$
\begin{aligned}
& G=\widetilde{\rho} k T+\frac{2 \pi}{3} \widetilde{\rho}^{2} c(1-c) \int d r^{\prime} r^{\prime 2} V\left(r^{\prime}\right) g^{e q}\left(r^{\prime}\right)- \\
& -\frac{2 \pi}{3} \widetilde{\rho}^{2} c(1-c) \int d r^{\prime} r^{\prime 3} V\left(r^{\prime}\right) \frac{d g\left(r^{\prime}\right)}{d r^{\prime}} .
\end{aligned}
$$

The stiffness of the bond is decreases, thus the temperature-dependence of the effective diffusion coefficient weakens. It is given by

$D_{\mathrm{eff}}=D\left\{1-\frac{T_{c}-a}{T}\right\}$ 
where

$a=\frac{2 \pi}{3} \widetilde{\rho}^{2} c(1-c) \int d r^{\prime} r^{\prime 3} V\left(r^{\prime}\right) \frac{d g\left(r^{\prime}\right)}{d r^{\prime}}$,

and

$T_{c}=-\frac{2 \pi}{3} \widetilde{\rho}^{2} c(1-c) \int d r^{\prime} r^{2} V\left(r^{\prime}\right) g^{e q}\left(r^{\prime}\right)$

is the critical temperature of the first-order phase transition accompanied by the formation of a spatial inhomogeneous structure in the particle distribution. The relevance of the correlation length is that it measures the range over which colloidal particles in the unsorted system are correlated. Since $\frac{d G}{d \widetilde{\rho}} \rightarrow 0$ the correlation length $\xi=\sqrt{S / \frac{d G}{d \widetilde{\rho}}}$ diverges under the approach to the critical point and also under the approach to the off-critical part of the spinodal decomposition. This means that at the critical point each colloidal particle in the system is correlated with all other colloidal particles. Hence the finite force is insufficient to break up these many-particle correlations and to generate the system flow since the viscosity diverges under the approach to the spinodal. The effective diffusion coefficient depends on the correlation length as $D_{\text {eff }}=D \xi^{-2}$, this observation illustrates the decrease of the relaxation time near the temperature the first-order phase transition.

In the case of structure ordering we have to take into account all the physical processes related to the structure formation, so we propose the following physical picture of the behavior of the effective diffusion coefficient: in course of formation of the liquid crystal each particle is dressed in an elastic deformation coat of the inhomogeneous director field distribution. This provides the increase of the effective mass of each particle and the decrease of the effective diffusion coefficient. In the course of liquid crystal formation particles interact due to the induced director field deformation. This repulsive interaction provides the increase of the collective effective diffusion coefficient. When the interaction generates the first-order phase transition accompanied by the inhomogeneous particle distribution the effective diffusion coefficient decreases. This effect is caused by the long-range correlations of particles, which are foreign in the liquid crystal. Within the context of this physical picture and with regard to the experimental data the behavior of the collective diffusion coefficient can be de- scribed by the approximation formula given by

$D_{\mathrm{eff}}=D_{0}\left[1-b\left(T-T_{\mathrm{in}}\right)^{2}\right]\left\{1-\frac{T_{c}-a}{T}\right\}$

where $T_{\text {in }}$ is the temperature of the phase transition in the medium in the liquid crystal state and $b$ is the coefficient that determines the dependence on the effective mass or friction drag of the moving foreign particles. This relation describes the dependence of the effective collective diffusion coefficient on temperature and particle density in a wide range of values of these quantities. To conclude we note that the behavior of the effective diffusion coefficient should take into account the particle dressing by the elastic deformation coat. This physical phenomenon provides the increase of the effective mass of each particle, enhances the drag force and decreases the effective diffusion coefficient. In the course of liquid crystal formation the interaction between particles is induced by the director field deformation. The interactions in the system can cause the first-order phase transition that also leads to the decrease of the effective diffusion coefficient. The formation of ordered structures is possible due to long-range correlations in many-particle systems. This physical picture makes it possible to interpret the collective diffusion processes observed experimentally.

This research was partially funded by the State Fund for Fundamental Research of Ukraine (Project $F$ 76/44-2018).

1. P. Poulin, H. Stark, T.C. Lubensky, D.A. Weitz. Novel colloidal interactions in anisotropic fluids. Science 275, 1770 (1997).

2. P. Poulin, V. Cabuil, D.A. Weitz. Direct measurement of colloidal forces in an anisotropic solvent. Phys. Rev. Lett. 79, 4862 (1997).

3. P. Poulin, V.A. Raghunathan, P. Richetti, D. Roux. On the dispersion of latex particles in a nematic solution. I. Experimental evidence and a simple model. J. Phys. I France 4, 1557 (1994).

4. P. Poulin, D.A. Weitz. Inverted and multiple nematic emulsions. Phys. Rev. E 57, 626 (1998).

5. I. Muševic, M. Škarabot, U. Tkalec, M. Ravnik, S. Žumer. Two-dimensional nematic colloidal crystals self-assembled by topological defects. Science 313, 954 (2006).

6. V. Nazarenko, A. Nych, B. Lev. Crystal structure in nematic emulsion. Phys. Rev. Lett 87, 075504 (2001).

ISSN 2071-0194. Ukr. J. Phys. 2019. Vol. 64, No. 1 
7. A. Nych, U. Ognysta, M. Skarabot, M. Ravnik, S. Zumer, I. Musevic. Assembly and control of 3D nematic dipolar colloidal crystals. Nature Communications 4, 1489 (2013).

8. T. Turiv, I. Lazo, A. Brodin, B.I. Lev, V. Reiffenrath, V.G. Nazarenko, O.D. Lavrentovich. Effect of collective molecular reorientations on Brownian motion of colloids in nematic liquid crystal. Science 342, 1351 (2013).

9. B.I. Lev, P.M. Tomchuk. Interaction of foreign macrodroplets in a nematic liquid crystal and induced supermolecular structures. Phys. Rev. E 59, 591 (1999).

10. K. Aoki, B.I. Lev, H. Yokoyama. Cluster formation of colloids in nematics. Mol. Cryst. Liq. Cryst. 367, 537 (2001).

11. B.I. Lev, S.B. Chernyshuk, P.M. Tomchuk, H. Yokoyama. Symmetry breaking and interaction of colloidal particles in nematic liquid crystals. Phys. Rev. E 65, 021709 (2002).

12. T. Ohtsuki, K. Okano. Diffusion coefficients of interacting Brownian particles. J. Chem. Phys. 77, 1443 (1982).

13. P. Prinsen, T. Odijka. Collective diffusion coefficient of proteins with hydrodynamic, electrostatic, and adhesive interactions. J. Chem. Rev. 127, 115102 (2007).

14. J.K. Dhont, G. Nagele. Critical viscoelastic behavior of colloids. Phys. Rev. E 58, 7710 (1998).

15. R. Verberg, I.M. de Schepper, E.G.D. Cohen. Viscosity of colloidal suspensions. Phys. Rev. E 55, 3143 (1997).

16. R.W. Ruhwandl, E.M. Terentjev. Monte Carlo simulation of topological defects in the nematic liquid crystal matrix around a spherical colloid particle. Phys. Rev. E 56, 5561 (1997).

17. R.W. Ruhwandl, E.M. Terentjev. Friction drag on a particle moving in a nematic liquid crystal. Phys. Rev. E 54, 5204 (1996).

18. E.D. Belockii, B.I. Lev, P.M. Tomchuk. Effective ion mass in a liquid crystal. JETP Lett. 31, 539 (1980).

19. H. Stark, D. Ventzki. Stokes drag of spherical particles in a nematic environment at low Ericksen numbers. Phys. Rev. E 64, 031711 (2001).
20. H. Stark, D. Ventzki. Non-linear Stokes drag of spherical particles in a nematic solvent. Europhys. Lett. 57, 60 (2003).

21. T.C. Lubensky, D. Pettey, N. Currier, H. Stark. Topological defects and interactions in nematic emulsions. Phys. Rev. E 57, 610 (1998).

22. S.B. Chernyshuk. High-order elastic terms, boojums and general paradigm of the elastic interaction between colloidal particles in the nematic liquid crystals. Eur. Phys. J. E 37, 6 (2014).

23. O.V. Kuksenok, R.W. Ruhwandl, S.V. Shiyanovskii, E.M. Terentjev. Director structure around a colloid particle suspended in a nematic liquid crystal. Phys. Rev. E 54, 5198 (1996).

24. E.M. Terentjev. Disclination loops, standing alone and around solid particles, in nematic liquid crystals. Phys. Rev. E 51, 1330 (1995).

25. H. Lowen. Solvent-induced phase separation in colloidal fluids. Phys. Rev. Lett. 74, 1028 (1995).

Received 17.08.18

\section{Б.І. Лев, А.Г. Загородній}

\section{КОЛЕКТИВНА ДИФУЗІЯ КОЛОЇДНИХ ЧАСТИНОК В РІДИННОМУ КРИСТАЛІ}

Р е 3 ю м е

Запропоновано теорію колективних дифузійних процесів у системі колоїдних частинок у рідинному кристалі. Описано особливості дифузії, які можна спостерігати експериментально. Знайдено залежність коефіцієнта дифузії від температури та густини частинок. Показано, що колективна дифузія в системі колоїдних частинок у рідинному кристалі пов'язана з пружними деформаціями директора, які зумовлюють далекосяжну взаємодію частинок. 